\title{
Pesquisas contextuais e seus desafios: uma contribuição a partir de investigações sobre arranjos espaciais em creches
}

\author{
Mara I. Campos-de-Carvalho \\ Universidade de São Paulo - Ribeirão Preto
}

\begin{abstract}
Resumo
Objetivando caracterizar pesquisas ecológicas, diferenciando-as de pesquisas não ecológicas, são apresentados pressupostos compartilhados por vários autores, apesar de suas divergências na análise da relação pessoa-ambiente (nas dimensões focalizadas, metodologia ou teoria). Uma breve visão introdutória da abordagem ecológica em psicologia é descrita. São discutidos dois critérios, interligados entre si e conectados aos pressupostos básicos, que diferenciam pesquisas ecológicas ou contextuais das não ecológicas ou não contextuais. Nossa experiência de condução de pesquisas contextuais, sobre a relação entre arranjo espacial e ocupação do espaço por crianças em creches, ilustra vários aspectos abordados; são descritos, resumidamente, três conjuntos de variáveis contextuais adicionais, estudadas para verificar se afetariam a ocupação espacial. São apresentados certos desafios metodológicos em pesquisas contextuais.
\end{abstract}

Palavras-chave: psicologia ambiental; abordagem ecológica; arranjo espacial; creche

\begin{abstract}
Contextual research and its challenges: the contribution of investigations on day care center spatial arrangements. The assumptions of the ecological research are presented, with the objective of characterizing the ecological research and its differentiation from nonecological research. These assumptions are shared by several authors, in spite of their divergences in the analysis of man-environment relationship (such as the ones on focused dimensions, methodology and theory). A brief view of the introduction of ecological ideas into psychology is given. Two criteria connected to the shared assumptions are useful to distinguish ecological and nonecological research. Our experience in carrying out contextual research on the relationship between spatial arrangement and the use of space by children in day care centers is used to illustrate many of the focused aspects; three groups of additional contextual variables are briefly presented, which are studied to examine their influence on spatial distribution. Some methodological challenges in contextual research are presented.
\end{abstract}

Keywords: environmental psychology; spatial arrangement; ecological approach; day care cente

$\mathrm{C}$ om o objetivo de caracterizar pesquisas contextuais ou ecológicas na Psicologia, apontando suas diferenças com pesquisas não contextuais, o presente artigo inicia-se apresentando uma breve visão da abordagem ecológica em Psicologia e seus pressupostos, encerrando-se com a exposição de uma série de desafios metodológicos que ainda se encontram sem resposta. Nossa experiência de pesquisa permeará nossas colocações, ilustrando, quando necessário, alguma característica de pesquisas contextuais.

Há mais de uma década, estamos pesquisando a relação entre arranjo espacial - uma variável física do contexto imediato, que diz respeito à maneira como móveis e equipamentos existentes em um local estão posicionados entre si (Legendre, 1986, 1999) - e a ocupação do espaço por grupos de crianças pequenas em creche. Analisamos a contribuição do arranjo espacial para a ocorrência das interações das crianças tanto entre si como com a(s) educadora(s). As interações entre crianças, em nosso ponto de vista, são tão importantes para o desenvolvimento infantil quanto as interações com adultos, ambas exigindo competências diversas da criança, servindo a diferentes funções (Hartup, 1987; Oliveira \& RossettiFerreira, 1993). Nossos estudos inserem-se na interface entre a Psicologia Ambiental e a do Desenvolvimento, cuja integração, embora necessária, não se faz sem desafios e dificuldades (Campos-de-Carvalho, no prelo).

Os resultados obtidos em nossas pesquisas (Camposde-Carvalho, 1989; Campos-de-Carvalho \& Mingorance, 1999; Campos-de-Carvalho \& Padovani, 2000; Campos-de-Carvalho \& Rossetti Ferreira, 1993; Meneghini \& Campos-de-Carvalho, 1997) têm mostrado a contribuição do arranjo espacial para a ocorrência de interações de coetâneos, tanto entre si como com a educadora, utilizando a metodologia denomina- 
da por Bronfenbrenner $(1977,1979)$ de experimento ecológico. Esta metodologia propõe a realização de manipulações sistemáticas de uma única variável, a que está sob investigação - em nossos estudos, o arranjo espacial -, mantendo-se inalterados os demais componentes ambientais presentes. Ou seja, aquelas manipulações são feitas no interior do sistema ecológico onde ocorre o fenômeno sob estudo, preservando-se, tanto quanto possível, o sistema de interdependência entre os componentes ambientais.

\section{Abordagem ecológica na Psicologia}

O termo ecologia diz respeito às inter-relações organismo-ambiente, tendo suas raízes lingüísticas na palavra grega oikos (casa, espaço de vida) e científicas na Biologia, com a Teoria da Evolução de Darwin. O termo foi cunhado e introduzido na ciência em 1873, pelo zoólogo alemão Ernest Haeckel (Tudge, Gray \& Hogan, 1997; Valsiner \& Benigni,1986). Diferentes perspectivas ecológicas desenvolveram-se em diversas disciplinas (por exemplo, na sociologia, geografia, antropologia, economia, saúde pública e em diversas áreas da psicologia), buscando compreender a natureza das inter-relações da pessoa e seu contexto (Stokols, 1992; Tudge, Gray \& Hogan, 1997; Valsiner \& Benigni, 1986).

Não temos como escopo traçar uma perspectiva histórica da introdução de idéias ecológicas na psicologia (ver Tudge, Gray \& Hogan, 1997; Valsiner \& Benigni, 1986). Entretanto, nas décadas de 1930 e 1940 tais idéias já estavam presentes, embora com conceituações diferentes entre os autores sobre as relações organismo-ambiente. Brunswick (1943, citado por Valsiner \& Benigni, 1986) procedia a uma análise separada do ambiente e do indivíduo, realizando um estudo correlacional e probabilístico de ambos. Outros pesquisadores propunham uma análise conjunta pessoa-ambiente, como Koffka e Lewin (ambos citados por Valsiner \& Benigni, 1986). Koffka (1935, citado por Stokols, 1987) manifestava interesse claro pelo contexto ecológico do comportamento e Lewin (1951/1965) já afirmava a interdependência entre as várias partes componentes do espaço de vida, conceito este que se refere ao ambiente percebido ou experienciado pela pessoa (Campos-de-Carvalho, 1993).

Nas décadas de 1950 e 1960, o comportamentalismo contribuiu para enfatizar o papel do ambiente, ao evidenciar como os processos de aprendizagem e de desenvolvimento dependiam de condições ambientais (Valsiner \& Benigni, 1986). Ademais, a "herança teórica de Koffka foi a base para a emergência (...) da psicologia ecológica como uma subdisciplina separada desde os anos de 1960" (Valsiner \& Benigni, 1986, p.206), tal como o foi, por exemplo, para a abordagem ecológica de Gibson sobre a percepção visual (Tudge, Gray \& Hogan, 1997).

Com a emergência da Psicologia Ambiental nos anos 1970 - a qual se propõe a estudar a relação bidirecional entre pessoa e ambiente, priorizando os aspectos físicos (Proshansky, Ittelson \& Rivlin, 1970) -, cresceu o interesse pelo uso do termo ecológico na psicologia. Stokols (1978) considera a Psicologia Ecológica como um dos vários domínios da Psico- logia Ambiental, citando os trabalhos de Barker (1965, 1968, 1969) como uma base para a emergência da psicologia ecológica, embora já existissem outras abordagens ecológicas na psicologia, anteriormente à emergência da Psicologia Ambiental, como vimos descrevendo. Pela perspectiva ecológica, o ambiente é concebido "em termos multidimensionais e molares, e o foco de análise está nas inter-relações entre pessoas e seus meios sócio-físicos [...] muito mais do que nas conexões entre estímulos discretos e respostas comportamentais...” (Stokols, 1978, p.254).

Finalizando este breve relato da introdução de idéias ecológicas na Psicologia, não poderíamos deixar de salientar os trabalhos de Barker e seus colaboradores (Barker, 1965, 1968, 1969). Seus estudos iniciaram-se anteriormente à emergência da Psicologia Ambiental, constituindo-se na primeira análise sistemática, em Psicologia, para demonstrar o impacto do ambiente ecológico no comportamento humano. A maioria dos outros pesquisadores ambientais, na mesma época, dirigia sua atenção não para o ambiente físico molar, mas sim para o espaço de vida de Lewin (1951/1965), ou então para os "estímulos micro-ambientais da psicologia perceptual e operante” (Stokols, 1978, p. 255). Barker foi aluno e colaborador de Lewin, porém diferenciou o ambiente psicológico, ou espaço de vida na terminologia de Lewin, do ambiente ecológico (que engloba os contextos objetivos e pré-perceptuais do comportamento), embora os dois tipos de ambiente façam parte de sua psicologia ecológica (Barker, 1968; Campos-deCarvalho, 1993; Carneiro \& Bindé, 1997). Barker (1965) propôs uma análise naturalística da relação ambiente-comportamento, cuja unidade de análise é o conceito de behavior setting; cada behavior setting tem uma realidade objetiva, com atributos físicos, espaciais e temporais, englobando um padrão extra-individual de comportamentos que são característicos daquela situação, constituindo-se em fenômenos comuns da vida diária (aula de matemática, jogo de futebol do time $\mathrm{A}$ e $\mathrm{B}$, missa de domingo às 8 horas, etc.).

Concluindo este tópico, embora as noções de ecologia já estivessem em uso na Psicologia desde a década de 1930, com a Psicologia Ambiental e, em especial, com a Psicologia Ecológica, tem havido um interesse e preocupação crescente com o termo ecológico (Stokols, 1987; Valsiner \& Benigni, 1986). A pesquisa ecológica (ou pesquisa contextualizada, ou pesquisa orientada ecologicamente) passou a ser vista, em várias áreas da Psicologia, como uma perspectiva alternativa, do ponto de vista metodológico e teórico, ao método científico tradicional. Este é visto como não apropriado para analisar o comportamento humano dentro do contexto em que ocorre, devido, por exemplo, à exclusão, no planejamento experimental, de variáveis com importância potencial para o fenômeno sob estudo (Altman \& Rogoff, 1987; Stokols, 1987).

O termo psicologia ecológica (ou outros termos sinônimos comumente encontrados, tais como perspectiva ecológica, abordagem ecológica, perspectiva contextual, abordagem contextual) vem sendo utilizado num sentido mais amplo que a Psicologia Ecológica de Barker, e em várias outras áreas da Psicologia, além da Psicologia Ambiental. Haja vista, por exemplo, as perspectivas ecológicas para o estudo do desen- 
volvimento humano (Bronfenbrenner, 1979, 1993; Bronfenbrenner \& Morris, 1998; Valsiner, 1987) e o campo da ecologia social que focaliza os contextos sociais, institucionais e culturais (Stokols, 1992).

Entretanto, existem certos pressupostos comuns às várias perspectivas ecológicas em Psicologia, que orientam as pesquisas contextuais, foco de nosso próximo tópico.

\section{Pesquisa contextual ou ecológica}

Abordaremos neste tópico: (1) alguns pressupostos básicos; (2) critérios diferenciadores de pesquisa ecológica e não ecológica; (3) variáveis contextuais.

\section{Alguns pressupostos básicos}

Apesar de haver diferenças nas dimensões focalizadas (variáveis físicas, sociais, culturais, psicológicas, etc.) e mesmo na metodologia (manipulativas e não manipulativas) ou teoria utilizada para analisar a relação pessoa-ambiente, há certos pressupostos básicos compartilhados por pesquisadores em Psicologia, adeptos de uma abordagem ecológica.

Em trabalhos anteriores (Campos-de-Carvalho, 1993, 1998, no prelo), já tivemos oportunidade para apresentar alguns pressupostos que caracterizam uma abordagem ecológica em Psicologia, tais como: (1) uma visão bidirecional da relação pessoa-ambiente, propondo que características da pessoa e do contexto interagem, modificando-se mutuamente; implícito está que não é apenas o ambiente que exerce influência na pessoa, mas esta também age e modifica o ambiente, embora haja uma tradição na Psicologia, até mesmo na Psicologia Ambiental, em examinar a primeira direção (Pinheiro, 1997); (2) interdependência de variáveis na explicação causal de fenômenos psicológicos, devido às influências recíprocas entre os componentes físicos e humanos de um dado contexto ambiental; (3) um contexto ambiental nunca é neutro, mesmo aquele cotidiano e habitual; qualquer um de seus aspectos influencia e é influenciado pelas pessoas usuárias daquele contexto; (4) unicidade do ambiente - há vários aspectos ambientais, tais como os físicos (objetos, equipamentos, características espaciais), os sociais (pessoas que utilizam aquele contexto, seus papéis, atividades, valores culturais) e os pessoais ou psicológicos (percepção do ambiente pelos participantes, suas expectativas, experiências anteriores); é apenas para finalidade de pesquisa e/ou intervenção que se extrai do ambiente um de seus aspectos, constituindo-se em diferentes maneiras de analisar a mesma situação.

Stokols (1987) aponta quatro pressupostos centrais às diversas perspectivas contextuais na psicologia, alguns similares aos que apresentamos e outros complementares: (1) visão do fenômeno psicológico em relação ao meio espacial, temporal e sócio-cultural no qual ele ocorre; (2) relevância de análises molares e longitudinais das atividades diárias da pessoa e seus contextos, especialmente em estudos focalizando respostas da pessoa a estímulos ambientais discretos ou a eventos de curto prazo; (3) procura por um equilíbrio entre a busca por leis e relações generalizáveis e uma análise da especificidade situacional do fenômeno psicológico; (4) tanto no planejamento de pesquisas como na decisão para aplicação de dados de pesquisas em intervenções na comunidade e para o desenvolvimento de políticas públicas, é necessário considerar, além da validade interna da pesquisa, a validade ecológica e externa.

A seguir, vamos abordar, sucintamente, apenas a validade ecológica, para não nos afastarmos de nosso objetivo. Entretanto, alertamos que um estudo ecológico, além da busca pela sua validade ecológica, deve se preocupar, como qualquer outra pesquisa, com sua validade interna. Parafraseando Bronfenbrenner (1977, p. 514), não há “uma dicotomia entre rigor e relevância” (para definição dos demais tipos de validade, ver Cook \& Campbell, 1979).

Não há uma conceituação única quanto à validade ecológica; ela é utilizada com referência a significados diversos, tais como o ambiente vivenciado pelos participantes de um estudo, como validade externa, ou validade transcontextual, ou validade de constructo (Bronfenbrenner, 1977, 1979; Campos-de-Carvalho, 1993; Hultsch \& Hickey, 1978; Petrinovitch, 1979; Scheidt, 1981; Stokols, 1987; Weisz, 1978). Apesar de fugir de nosso escopo uma apresentação aqui destas várias conceituações, é relevante apontar que, embora tenha significados diversos, há um consenso entre os autores de que o fato de uma pesquisa ser conduzida em situação de vida diária não lhe confere, automaticamente, validade ecológica. Nenhuma situação de pesquisa é designada, à priori, como válida ou não, pois isso depende da questão que está sendo investigada; ou seja, dependendo do problema da pesquisa, o laboratório pode ser visto como um contexto ecológico. Bronfenbrenner $(1977,1979)$ nos dá um exemplo claro: para um estudo cujo objetivo seja investigar a interação entre mãe e criança quando esta é colocada numa situação não familiar, o laboratório se constitui em um ambiente mais apropriado que certas situações de vida cotidiana (Bronfenbrenner, 1977, 1979). Por outro lado, algumas situações ditas naturais podem ter um caráter tão excepcional ou atípico para certas pessoas, que não se constituirão como representativas do fenômeno sob investigação (Legendre, 1985). Em suma, o termo ecológico não é sinônimo de naturalístico (Valsiner \& Benigni, 1986).

Do nosso ponto de vista, para que uma pesquisa tenha validade ecológica, dentre outros critérios a seguir especificados, é necessário que seja conduzida em um contexto ambiental representativo do fenômeno sob estudo. Considerando a proposição de nossos estudos, para investigar o comportamento social (interações) de crianças pequenas quando em grupo, as creches se constituem numa realidade social representativa das experiências coletivas das crianças, constituindo-se, então, num contexto ecológico apropriado àquela questão.

\section{Critérios diferenciadores de pesquisas ecológicas e não ecológicas}

Conectados aos pressupostos básicos que orientam a condução de pesquisas ecológicas, dois critérios permitem diferenciá-las de pesquisas não ecológicas ou não contextuais, de acordo com Valsiner e Benigni (1986), cujo 
ponto de vista é compartilhado por outros autores (Bronfenbrenner, 1977, 1979; Bronfenbrenner \& Morris, 1998; Stokols, 1987). Tais critérios são interligados, sendo apresentados separadamente apenas por razões didáticas.

Primeiro critério - Desde que o fenômeno sob estudo está imerso em um sistema de interdependência de variáveis ou eventos contextuais, sendo influenciado por esse sistema (e o influenciando, através dos comportamentos das pessoas), a pesquisa ecológica, necessariamente, inclui esses eventos contextuais em sua análise do fenômeno. Enquanto que uma perspectiva não ecológica exclui estas variáveis de seu planejamento, especialmente na metodologia científica tradicional, segundo a qual manipula-se apenas uma variável, eliminando, tanto quanto possível, as demais variáveis que poderão interferir no fenômeno sob estudo; isto porque são tidas como "ruídos" que obscurecem a verdadeira natureza do fenômeno em questão.

A metodologia do experimento ecológico (Bronfenbrenner, 1977, 1979), utilizada em nossas pesquisas, contempla este critério, buscando preservar a complexidade do sistema ecológico na análise de um de seus componentes, o arranjo espacial e sua relação com a ocupação do espaço pelas crianças. Nossas manipulações do arranjo espacial são feitas no interior do sistema ecológico onde ocorre o fenômeno sob estudo, ou seja, no local e horário habitual de encontro diário do grupo de crianças e sua educadora (já familiarizadas entre si) para a ocorrência de atividades livres; a coleta de dados somente ocorre se houver a presença da educadora e de pelo menos $70 \%$ das crianças do grupo. Ao lado disso, tentamos manter presentes os demais componentes ambientais, tais como: utilização de materiais da própria creche e de uso habitual pelo grupo; a duração das sessões é de responsabilidade da educadora, tal como o é em sua rotina de trabalho; a coleta de dados é feita sem a presença do pesquisador e do operador das câmeras (fotográficas ou de vídeo), as quais funcionam automaticamente. Outros cuidados metodológicos são seguidos: camuflagem das câmeras, que são cobertas com pano (com exceção das lentes); presença constante dos suportes de madeira nas paredes (nos quais as câmeras são encaixadas durante a coleta de dados), cobertas pelos panos; coleta de dados na presença do arranjo habitual do espaço, antes de ser manipulado; período de familiarização do grupo aos novos arranjos, antes de coletar os dados, etc.

Segundo critério - Dada a concepção bidirecional, ou transacional, da relação pessoa-ambiente, a interação fenômeno investigado-contexto torna-se objeto de estudo e a inclusão desse processo de interação em uma pesquisa empírica é necessária para ser considerada como uma investigação ecológica. Em uma pesquisa não ecológica, o contexto pode até ser considerado importante, porém não é estudado diretamente, à medida que o fenômeno sob estudo interage com ele. Além disso, em estudos não contextuais, geralmente, há atribuição de uma fonte única de causalidade, enquanto que em pesquisas contextuais há um sistema causal inter-relacio- nado, incluindo a interdependência tanto de aspectos do organismo como do ambiente.

A prática tradicional em Psicologia de usar a análise de variância é um bom exemplo não ecológico. Por esta análise, sinteticamente aqui apresentada, "diferentes porções da variância são ‘causadas’ por diferentes 'efeitos' ou por suas interações” e presume-se que há a mediação de algum processo psicológico entre a manipulação de variáveis independentes e suas conseqüências, as variáveis dependentes, processo esse geralmente não especificado. Entretanto, não há informação explícita sobre "como" estes fatores que interagem se relacionam na realidade. A análise da variância, então, abarca a relação pessoa-ambiente simultaneamente, mas separadamente um do outro, pois o processo real de interação não foi focalizado na coleta de dados; ou seja, a interação pessoa-ambiente é eliminada do estudo empírico, não fazendo parte do planejamento experimental, faltando assim uma base empírica sobre como "esses fatores realmente interagiram" (Valsiner \& Benigni, 1986, p. 214). É este aspecto que caracteriza o estudo como não contextual, ou não ecológico.

Outro exemplo da tradição não ecológica na Psicologia é o uso de testes psicológicos, pois se analisa o fenômeno (inteligência, personalidade) em relação apenas às respostas dadas pela pessoa (por exemplo, certa ou errada, em um teste de inteligência), independentemente tanto do contexto de processos psicológicos que a pessoa utiliza para chegar àquela resposta, como da situação real de teste (Valsiner \& Benigni, 1986).

As nossas pesquisas contemplam este critério, havendo coleta e análise de dados específicas sobre a relação arranjo espacial e comportamentos infantis. Os dados são coletados diretamente no local de ocorrência de atividades livres das crianças. O procedimento inclui tantas fases quantas forem necessárias para manipulação da variável sob análise, havendo pelo menos duas sessões de coleta de dados em cada fase. Na maioria de nossos estudos, a primeira fase ocorre na presença do arranjo espacial habitual do local onde ocorrem atividades livres, geralmente caracterizado por um espaço central vazio e pela ausência de zonas circunscritas. Zonas circunscritas são áreas delimitadas, pelo menos em três lados, por barreiras baixas, estruturadas por mobiliários, parede, desnível do solo, etc.. Para transformar este arranjo habitual em um arranjo com zonas circunscritas, sempre houve a necessidade da introdução de estantes baixas de madeira, a serem usadas na delimitação das zonas, dada a escassez ou mesmo ausência de móveis. Estas estantes são introduzidas numa etapa intermediária (da qual coletamos e analisamos os dados) e colocadas nas laterais do local, para manter a mesma característica espacial do arranjo habitual (espaço central vazio e ausência de zonas circunscritas). A coleta de dados se inicia após cerca de 12 dias úteis de familiarização do grupo às estantes; tal precaução metodológica é necessária visto que nosso interesse não está na reação imediata das crianças às transformações realizadas. Na fase seguinte, duas zonas circunscritas são estruturadas aproveitando, sempre que possível, a quina de duas paredes para delimitar dois lados, sendo os outros dois estruturados por estantes baixas, ha- 
vendo uma pequena abertura para a passagem de crianças. A Figura 1 mostra fotos da sala de um grupo de crianças de 2-3 anos, ilustrando as três fases do estudo.

\section{Variáveis contextuais}

Stokols (1987) apresenta dois passos a serem seguidos em uma pesquisa contextual. Tal como num estudo não ecológico, a primeira tarefa é a identificação do fenômeno central a ser investigado (em nosso caso é a relação entre arranjo espacial e interações de crianças em creches) e das variáveisalvos a serem examinadas, as quais incluem a variável de predição e a variável de produto, ou variável conseqüência (em nossas pesquisas são, respectivamente, o arranjo espacial e a ocupação do espaço pelas crianças). Enquanto uma pesquisa não ecológica focalizaria apenas a relação entre estas duas variáveis (por exemplo, hipotetizando que a ocupação do espaço é conseqüência apenas do tipo de arranjo espacial), o pesquisador contextual deveria se indagar se esta relação poderia ser afetada e qualificada por outros aspectos ou variáveis contextuais que, em nosso caso, cercam o padrão de ocupação espacial, ou seja, variáveis conectadas no tem- po e no espaço com a maneira das crianças ocuparem o espaço.

Em outras palavras, a pesquisa contextual engloba variáveis contextuais adicionais às variáveis-alvos, as quais, provavelmente, qualificam a relação entre as duas variáveis-alvos. Segundo Stokols (1987), o segundo passo do pesquisador contextual, então, seria "definir um conjunto de variáveis contextuais ou situacionais que se pensa que exerçam uma influência importante sobre a forma e ocorrência do fenômeno alvo” (p. 43), incorporando medidas destas variáveis adicionais em sua análise contextual do fenômeno. Desta maneira, uma perspectiva contextual amplia o escopo de análise das variáveis sob investigação.

Aquele autor aponta ainda que a seleção destas variáveis contextuais para uma análise empírica pode ocorrer de um modo exploratório e ateórico ou pode ser guiada por uma teoria contextual com pressupostos sobre o fenômeno alvo, sendo esta última forma mais eficaz e sistemática. Uma teoria contextual explicitamente já prediz uma mudança na relação entre as variáveis-alvos, dependendo da presença ou ausência de certos fatores situacionais, explicando os porquês das

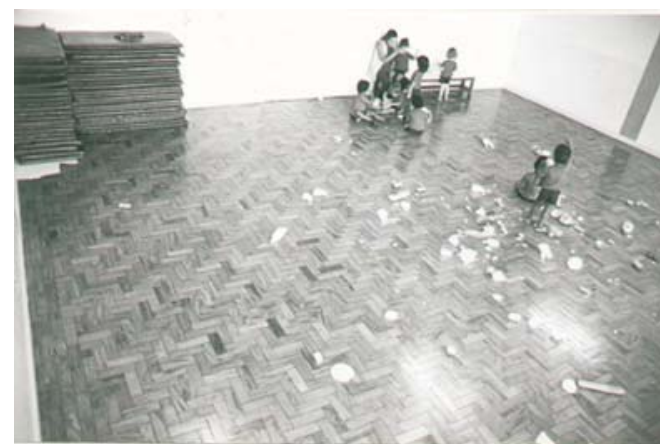

Fase 1 - Arranjo espacial habitual

(ausência de zona circunscrita)

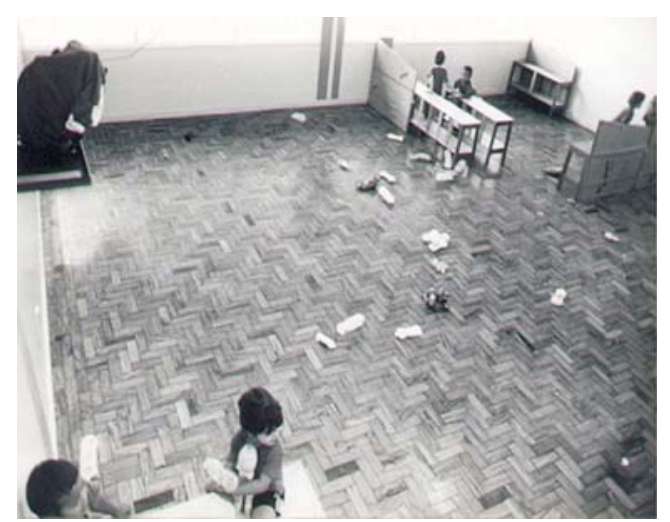

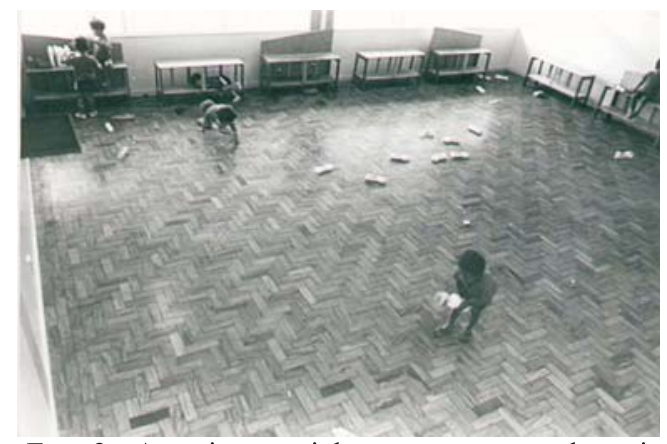

Fase 2 - Arranjo espacial com estantes nas laterais (ausência de zona circunscrita)

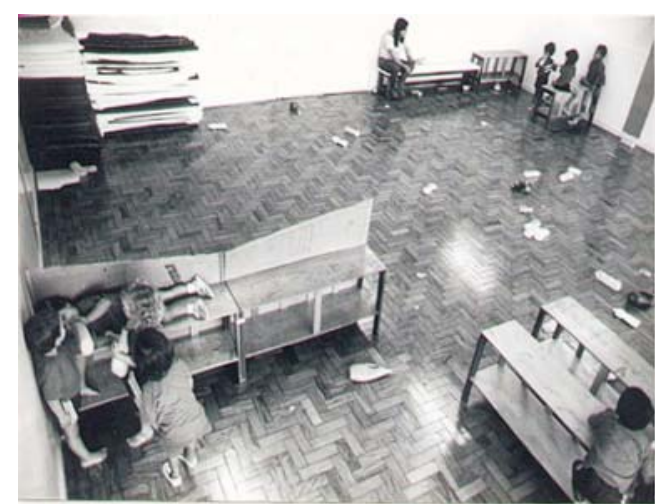

Fase 3 - Arranjo espacial com duas zonas circunscritas (longe e perto da educadora)

Figura 1. Fotos ilustrativas do arranjo espacial da sala de um grupo de crianças de 2-3 anos, em cada fase da coleta de dados. 
variações no fenômeno-alvo, o que, então, a diferencia de uma teoria não contextual. Na ausência de teorias contextuais, geralmente a identificação de fatores contextuais importantes ocorre através de avaliações posteriores, muitas vezes ao acaso, de uma maneira não programada, à medida que os pesquisadores comparam seus dados advindos de estudos conduzidos independentemente. Por exemplo, as primeiras formulações sobre as reações humanas à super-população e barulho foram tratadas de uma maneira não contextualizada, sendo os estudos empíricos planejados para testar hipóteses universais; entretanto, os resultados obtidos eram complexos, revelando diferenças marcantes nas reações, dependendo dos contextos situacionais; daí então, formulações contextuais foram desenvolvidas para explicar os resultados diversos e muitas vezes contraditórios.

De acordo com nossa experiência de condução de pesquisas contextuais sobre o arranjo espacial, a escolha de variáveis adicionais a serem analisadas advém de estudos anteriores, ou seja, ao término de uma análise específica, selecionamos outras a serem investigadas, seja através de nova coleta de dados ou através de novas análises dos dados já colhidos. Vemos que esta forma de escolha, embora não se baseie em uma teoria contextual (por falta desta) sobre o fenômeno alvo (relação entre arranjo espacial e interações de crianças em creches), está sendo útil para revelar fatores contextuais que influenciam e que não influenciam as variáveis-alvos.

Temos analisado, numa série de estudos, três conjuntos de variáveis adicionais, examinando sua influência na ocupação do espaço. Verificamos a preferência das crianças por determinadas áreas espaciais presentes simultaneamente no local, através da análise da localização espacial de cada criança a cada minuto, tendo por base a técnica de mapeamento comportamental (Elali, 1997; Ledingham \& Chappus, 1986; Proshansky, Ittelson \& Rivlin, 1970; Sommer \& Sommer, 1997).

Primeiro conjunto - estudos sobre as variáveis do contexto imediato (Campos-de-Carvalho \& Mingorance, 1999; Campos-de-Carvalho \& Rossetti-Ferreira, 1993):

(1) localização e tamanho da zona circunscrita (ZC), havendo uma ZC com maior área e estruturação, localizada longe da área em torno do local habitual da educadora e outra ZC menor e perto da área do adulto - houve ocupação preferencial pela ZC mais estruturada e longe do adulto;

(2) manipulação da variável circunscrição, com presença simultânea de três áreas espaciais formadas por estantes baixas, sendo uma zona circunscrita (ZC) e duas áreas espaciais sem circunscrição (estantes encostadas linearmente na parede) - houve ocupação preferencial da ZC;

(3) manipulação da variável superfície de apoio, havendo uma ZC cujas estantes delimitadoras tinham superfície de apoio $(30 \mathrm{~cm})$ e outra ZC com divisória sem superfície de apoio (5 cm) - ocupação preferencial da ZC com superfície de apoio;

(4) número crescente de ZC inter-fases (primeira fase, 1 ZC / fase seguinte, 2 ZCs / última fase, 3 ZCs) - com maior número de ZCs, houve um decréscimo significativo na ocupação da área do adulto e maior ocupação das ZCs.

Segundo conjunto - estudos sobre variáveis da situação de interação:

(1) análise da relação entre tamanho dos agrupamentos entre crianças e ocupação do espaço (Meneghini \& Camposde-Carvalho, 1997) - houve aumento no número de agrupamentos entre crianças e redução daqueles com a educadora em arranjos com número maior de ZCs; nas áreas mais estruturadas, específicas a cada fase, houve maior ocorrência de agrupamentos, especialmente aqueles com três ou mais crianças;

(2) impacto do arranjo espacial na ocupação do espaço por agrupamentos preferenciais, cujos componentes se associam mais freqüentemente, e os não-preferenciais, com pouca associação entre seus componentes (Campos-de-Carvalho \& Padovani, 2000) - evidenciou-se maior suporte do arranjo espacial para os agrupamentos não-preferenciais, embora seja relevante para os dois tipos de agrupamentos.

Terceiro conjunto - estudos sobre variáveis pessoais:

(1) verificação se crianças classificadas em diferentes subgrupos de interação, de acordo com sua freqüência de interação com as demais crianças, ocupam diferentemente o espaço quando em interação, em atividade individual, ao observar atentamente outras (espectador) e dirigir-se socialmente aos outros - o subgrupo de alta freqüência interacional destacou-se por exibir menor ocorrência de atividade individual e do comportamento de espectador, não exibindo nenhuma interação na área do adulto, na fase com maior número de ZCs; visando o aumento da ocorrência de interações entre crianças, o papel de suporte do arranjo espacial com maior número de ZCs foi mais evidente para o subgrupo com menor competência social (Meneghini \& Campos-de-Carvalho, 2000).

(2) extensão da análise da relação entre arranjo espacial e ocupação do espaço para as idades adjacentes (1-2 e 3-4 anos) à faixa etária de 2-3 anos, anteriormente focalizada em nossas pesquisas e nos estudos de Legendre (1985, 1986, 1999) - tal como em estudos anteriores, observou-se que diferentes arranjos espaciais levam a padrões diversos de distribuição espacial, sendo que o padrão de ocupação espacial das crianças de 3-4 anos é muito similar ao das crianças de 23 anos; as de 1-2 anos apresentam um padrão diferente quando na presença de arranjo com zonas circunscritas, pois ocupam com freqüência semelhante as ZCs e a área ao redor do adulto, indicando que crianças menores necessitam mais da proximidade da educadora do que as mais velhas (Bomfim \& Campos-de-Carvalho, no prelo).

Nestes dois últimos estudos citados, investigamos duas características pessoais (competência social e idade), em resposta à afirmação de Bronfenbrenner (1993), a qual vem chamando nossa atenção já há algum tempo (Campos-de-Carvalho, 1993). Ele aponta que a maioria dos estudos sobre as propriedades do contexto desconsidera as características 
pessoais de seus usuários, como se todas as pessoas fossem igualmente afetadas pelo ambiente.

Ao considerarmos o pressuposto da unicidade do ambiente, já apontamos anteriormente que há vários aspectos ambientais e um deles diz respeito ao aspecto pessoal ou psicológico. Desta maneira, o fato de investigarmos duas características ou aspectos pessoais (competência social e idade), não implica, necessariamente, numa descaracterização da pesquisa como contextual ou ecológica, pois vemos qualquer contexto ambiental como um sistema de interdependência entre seus componentes físicos e humanos. Entretanto, este nosso ponto de vista contraria, até certo ponto, a visão de Stokols (1987), pois, embora ele se refira a "teorias” contextuais (e não pesquisas), ele afirma que o foco das mesmas está "muito mais em moderadores situacionais do que nos moderadores intra-pessoais das relações ambiente-comportamento” (p. 45). Tal afirmação pode levar à percepção de que uma pesquisa contextual não deveria focalizar variáveis ou características pessoais, o que não é o nosso ponto de vista.

Esta nossa visão é muito influenciada por Bronfenbrenner, provavelmente porque trabalhamos na interface entre a Psicologia do Desenvolvimento e a Ambiental. Com base em uma perspectiva sistêmica e ecológica, nossa visão de desenvolvimento humano enfatiza a relação bidirecional e interdependente entre pessoa-ambiente. Desenvolvimento implica em processos através dos quais características da pessoa e do ambiente interagem, produzindo tanto continuidade quanto mudança nas características da pessoa durante o curso de vida, no modo como a pessoa percebe e negocia com seu ambiente; isto é, mudança e constância nas capacidades da pessoa em descobrir, manter ou alterar as propriedades do ambiente, as quais, por sua vez, também exercem influência nas características da pessoa (Bronfenbrenner, 1993; Bronfenbrenner \& Morris, 1998).

Finalizamos este tópico sobre pesquisas ecológicas e não ecológicas, considerando se todo fenômeno psicológico seria ou não passível de uma análise contextual ou ecológica. Stokols (1987) aponta que pode haver certos fenômenos psicofisiológicos que são relativamente invariantes através de várias situações, levando em conta os aspectos sociais e físicos do contexto. Por exemplo, há processos farmacológicos que são bem mais dependentes de fatores intra-pessoais e/ ou do tipo de droga ingerida, do que de aspectos físicos e sociais do contexto; desta maneira, tais processos, de acordo com Stokols, não se adequariam a uma análise contextual. Concordamos parcialmente com tal afirmação, quanto à busca de variáveis físicas ou sociais do ambiente relacionadas àqueles processos psicológicos. Mas, em nosso ponto de vista, uma análise contextual seria aplicável, desde que o interesse estivesse em investigar os diversos fatores intra-pessoais que poderiam afetar, ou não, aqueles processos farmacológicos (especialmente considerando o ser humano e seus aspectos intra-pessoais como um dos componentes do ambiente, como já dissemos). Um segundo exemplo de Stokols diz respeito às pesquisas sobre barulho, com intensidade extremamente alta. Este fenômeno alvo não se adequaria a uma análise contextual, pois há vários estudos demonstrando que, acima de um certo limiar de intensidade do barulho, o impacto de fatores ambientais torna-se relativamente uniforme através de diferentes pessoas e situações.

Assim sendo, segundo Stokols (1987), qualquer fenômeno pode ser analisado contextualmente, desde que haja razões suficientes para se supor a mediação de fatores situacionais nas influências de condições ambientais sobre os comportamentos ou sobre a saúde das pessoas. A decisão, portanto, para se adotar, ou não, uma perspectiva ecológica para um dado problema de pesquisa, segundo Stokols, depende das evidências empíricas já existentes sobre a variabilidade do fenômeno através de diferentes situações (adequado para uma análise contextual), ou sobre a sua estabilidade (não adequado a uma análise contextual). Ademais, tal decisão depende também de considerações de ordem prática, tais como disponibilidade pessoal e de tempo do pesquisador e de financiamentos suficientes para conduzir estudos empíricos sobre o fenômeno alvo, através de diferentes contextos ambientais, o que nos remete ao último tópico deste artigo.

\section{Limitações e desafios metodológicos}

Em artigos anteriores (Campos-de-Carvalho, 1993; 1998), indicamos duas limitações metodológicas ao se utilizar uma abordagem ecológica, as quais apresentaremos resumidamente aqui.

Considerando a interdependência de variáveis na explicação causal de fenômenos psicológicos, é impossível, do ponto de vista metodológico, analisar simultaneamente vários aspectos ambientais que afetam aquele fenômeno sob estudo. Por necessidade metodológica, tem-se que selecionar um aspecto ambiental para estudo, porém preservando o sistema de interdependência entre os componentes ambientais, especialmente durante a interpretação dos dados obtidos.

Em segundo lugar, conforme a questão sob investigação, o planejamento de pesquisa irá priorizar o ambiente afetando o comportamento observado, ou este influenciando o ambiente. Dificilmente um planejamento de pesquisa conseguiria abarcar simultaneamente as duas direções, apesar do pressuposto de que ambos, ambiente e pessoa, modificam-se mutuamente. Em nossas pesquisas, por exemplo, priorizamos a influência do arranjo espacial na ocupação do espaço pelas crianças.

É, então, em decorrência de limitações metodológicas que um planejamento de pesquisa ecológica separa, aparentemente, o fenômeno psicológico do contexto no qual ele ocorre. No entanto, como diz Valsiner (1987), esta é uma "separação inclusiva” (p.17) da pessoa em relação ao seu ambiente, pois o contexto ambiental entra necessariamente na análise do fenômeno estudado. Mesmo que o foco priorizado seja o fenômeno, a importância do contexto é reconhecida no estudo e vice-versa, ou seja, se o foco é o contexto, a relevância da influência do comportamento sobre o ambiente deve ser reconhecida. O pesquisador que adota uma abordagem ecológica está consciente de tais limitações ou simplificações do planejamento de pesquisa. É relevante que torne claro os pressupostos por ele assumidos quanto à concepção das 
relações pessoa-ambiente e que evite uma explicação unidirecional quanto à influência daquele aspecto ambiental selecionado sobre o fenômeno investigado, o que seria assumir uma causalidade elementarista e não sistêmica, na linguagem de Valsiner (1987).

Entretanto, apesar do encanto que nos traz uma concepção transacional da relação pessoa-ambiente, pela proposta de análises amplas, ela também traz muitas incertezas e desafios metodológicos, ainda hoje atuais, reunidos nas seguintes questões (Altman \& Rogoff, 1987; Stokols, 1987; Valsiner, 1987; Valsiner \& Benigni, 1986):

(1) como construir uma teoria de fenômenos mutáveis e holísticos?

(2) quais métodos usar para estudar estes fenômenos?

(3) como incorporar mudança e fatores temporais aos fenômenos psicológicos, especialmente considerando que a Psicologia, tradicionalmente, estuda-os estaticamente (até mesmo a Psicologia do Desenvolvimento, supostamente com interesse em fatores temporais e mudança)?

(4) quais aspectos diferenciam a pesquisa contextual da não contextual?

(5) uma análise contextual é aplicável a todos os fenômenos psicológicos? Ou seja, para quais fenômenos se justifica uma análise contextual e para quais não se justifica?

(6) como traçar os limites contextuais de um fenômeno e quais critérios utilizar para decidir quais variáveis contextuais farão parte da análise?

Como muito bem afirmado por Stokols (1987), é de extrema necessidade transformar o ponto de vista transacional em estratégias operacionais, tanto para pesquisas como para desenvolvimento teórico. Todos estes questionamentos apontam os desafios atuais, ao se utilizar uma perspectiva ecológica ou contextual, para os quais, cada vez mais, se faz urgente buscar respostas, para podermos avançar tanto na condução de pesquisas, como na construção de teorias contextuais.

Encerramos este artigo apontando, em relação às nossas pesquisas, a limitação que sentimos quanto à omissão de uma teoria contextual consistente para interpretação de nossos dados. Temos utilizado três propostas teóricas, embora cada uma delas focalize, para análise, aspecto(s) específico(s) da relação pessoa-ambiente: a perspectiva sistêmica de Urie Bronfenbrenner (1977; 1993; Bronfenbrenner \& Morris, 1998), denominada Ecologia do desenvolvimento humano; o conceito de apropriação do espaço (Moreno \& Pol, 1999; Pol, 1996) e a perspectiva teórico-metodológica da Rede de Significações (Rossetti-Ferreira, Amorim \& Silva, 2000).

Não cabe discutir no presente artigo a maneira como as usamos. Temos tentado buscar as convergências possíveis entre as três propostas; eventuais divergências entre elas, que não nos parecem incompatibilidades, não impedem a interação destes três pontos de vista, na busca de explicação teórica para os dados empíricos obtidos em nossos estudos (Campos-de-Carvalho, Bomfim \& Souza, no prelo).

Apesar da ausência de uma teoria contextual, a semelhança dos resultados de nossas pesquisas, obtidos em diferentes creches e, em especial, a semelhança entre nossos resultados e os de Legendre em creches parisienses, com crianças na mesma faixa etária (2-3 anos), em locais com o mesmo tipo de arranjo espacial, porém diferindo em muitos outros aspectos, evidencia a forte interdependência entre arranjo espacial e ocupação do espaço. Este aspecto contribui para a generalização de nossos resultados para outros contextos educacionais coletivos, uma dentre as dificuldades apontadas na abordagem ecológica (Weisz, 1978).

Do ponto de vista prático - uma das especificidades de pesquisas na área da Psicologia Ambiental é a sua contribuição para a resolução de problemas da comunidade (Stokols, 1995) -, nossos estudos têm indicado a relevância do arranjo espacial no planejamento de ambientes coletivos para préescolares, favorecendo não só as interações entre crianças, mas também delas com a educadora, contribuindo para a melhoria da qualidade do atendimento coletivo de crianças pequenas. Através de manipulações pouco custosas, mesmo em termos financeiros, a educadora, ao estruturar o espaço com zonas circunscritas, promove interações entre crianças sem a sua intermediação direta; isto a torna muito mais disponível para estabelecer contato com uma criança individualmente ou com um subgrupo.

\section{Agradecimentos}

Agradecimentos à FAPESP e ao CNPq pelos auxílios recebidos às pesquisas que subsidiam este trabalho.

\section{Referências}

Altman, I., \& Rogoff, B. (1987). World views in psychology: Trait, interactional, organismic, and transactional perspectives. In D. Stokols \& I. Altman (Orgs.), Handbook of Environmental Psychology (vol. I, pp. 5-40). Nova York: John Wiley.

Barker, R. G. (1965). Explorations in ecological psychology. American Psychologist, 20, 1-14.

Barker, R. G. (1968). Ecological psychology. Palo Alto: Stanford University Press.

Barker, R. G. (1969). Wanted: An eco-behavioral science. In E. P. Willems \& H. I. Raush (Orgs.), Naturalistic viewpoints in psychological research (pp. 3143). Nova York: Holt, Rinehart \& Winston.

Bomfim, J. A. O., \& Campos-de-Carvalho, M. I. (no prelo). Arranjos espaciais e ocupação do espaço por crianças de 1-2 e 3-4 anos em creches. In Departamento de Psicologia e Educação, F.F.C.L.R.P./USP (Org.), Artigos do V Seminário de Pesquisa (Tomo II) do Programa de Pós-Graduação em Psicologia. Ribeirão Preto: Autor.

Bronfenbrenner, U. (1977). Toward an experimental ecology of human development. American Psychologist, 32, 513-531.

Bronfenbrenner, U. (1979). The ecology of human development. Cambridge: Harvard University Press.

Bronfenbrenner, U. (1993). The ecology of cognitive development: research models and fugitive findings. In R. H. Wozniack \& K. W. Fischer (Orgs.), Development in context: acting and thinking in specific environments (pp. 344). New Jersey: Erlbaum.

Bronfenbrennner, U., \& Morris, P. A. (1998). The ecology of developmental processes. In W. Damon \& R. M. Lerner (Orgs.), Handbook of child psychology (Vol. I, pp. 993-1028). Nova York: Wiley.

Campos de Carvalho, M. I. (1989). Organização espacial da área de atividades livres em creches [artigo completo]. In Sociedade de Psicologia de Ribeirão Preto (Org.), Anais da $18^{\underline{a}}$ Reunião Anual de Psicologia (pp. 305-310). Ribeirão Preto: Autor. 
Campos-de-Carvalho, M. I. (1993). Psicologia Ambiental - algumas considerações. Psicologia: Teoria e Pesquisa, 9(2), 435-447.

Campos-de-Carvalho, M. I. (1998). Comportamentos de crianças pequenas em creches e arranjo espacial. Temas em Psicologia - Sociedade Brasileira de Psicologia, 6(2), 125-133.

Campos-de-Carvalho, M. I. (no prelo). Psicologia ambiental e do desenvolvimento: o espaço em instituições infantis. In R. S. L. Guzzo, J. Q. Pinheiro \& H. Gunther (Orgs.), Psicologia Ambiental: entendendo as relações do homem com seu ambiente. Campinas, SP: Alínea.

Campos-de-Carvalho, M. I, Bomfim, J. A., \& Souza, T. N. (no prelo). Organização de ambientes infantis coletivos como contexto de desenvolvimento. In M. C. Rossetti-Ferreira, A. M. A. Carvalho, K. S. Amorim \& A. P. S. Silva (Orgs.), Rede de significações: uma nova perspectiva teórico-metodológica (capítulo 9). Porto Alegre, RS: ArtMed.

Campos-de-Carvalho, M. I., \& Mingorance, R. C. (1999). Zonas circunscritas e ocupação do espaço por crianças pequenas em creche. Revista Interamericana de Psicologia, 33(2), 67-89.

Campos-de-Carvalho, M. I., \& Padovani, F. H. P. (2000). Agrupamentos preferenciais e não preferenciais e arranjos espaciais em creches. Estudos de Psicologia, 5(2), 443-468.

Campos-de-Carvalho, M. I., \& Rossetti Ferreira, M. C. (1993). Importance of spatial arrangements for young children in day care centers. Children's Environments, 10(1), 19-30.

Carneiro, C., \& Bindé, P. J. (1997). A psicologia ecológica e o estudo dos acontecimentos da vida diária. Estudos de Psicologia, 2(2), 363-376.

Cook, T. D., \& Campbell, D. T. (1979). Quasi-experimentation: design and analysis issues for field settings. Boston: Houghton Mifflin.

Elali, G. A. (1997). Psicologia e Arquitetura: em busca do locus interdisciplinar. Estudos de Psicologia, 2(2), 349-362.

Hartup, W. W. (1987, julho). Relations and growth of social competence. In International Society for the Study of Behavioural Development (Org.), China Satellite Conference. Beijin, China: Autor.

Hultsch, D. F., \& Hickey, T. (1978). External validity in the study of human development - theoretical and methodological issues. Human Development, 21, 76-91.

Ledingham, J. E., \& Chappus, F. T. (1986). Behavioral mappings of children's social interactions: the impact of the play environment. Canadian Journal of Research in Early Childhood Education, 1, 137-148.

Legendre, A. (1985). L'experimentation écologique dans l'approche des comportements sociaux des jeunes enfants en groupes. In P. M. Baudonnière (Org.), Etudier l'enfant de la naissance à 3 ans (pp. 165-181). Paris: Collection Comportements, CNRS

Legendre, A. (1986). Effects of spatial arrangements on child/child and child/ caregivers interactions: an ecological experiment in day care centers [artigo completo]. In Sociedade de Psicologia de Ribeirão Preto (Org.), Anais da $16^{a}$ Reunião Anual de Psicologia (pp. 131-142). Ribeirão Preto, SP: Autor.

Legendre, A. (1999). Interindividual relationships in groups of young children and susceptibility to an environmental constraint. Environment and Behavior, 31(4), 463-486.

Lewin, K. (1965). Teoria de campo em ciência social. São Paulo: Edusp (tradução brasileira do original norte-americano de 1951).
Meneghini, R., \& Campos-de-Carvalho, M. I. (1997). Arranjos espaciais e agrupamentos de crianças pequenas em creche. Revista Brasileira de Desenvolvimento e Crescimento Humano, 7(1), 63-78).

Meneghini, R., \& Campos-de-Carvalho, M. I. (2000). Relação entre áreas espaciais e interação de crianças pequenas em creche. In Departamento de Psicologia e Educação, F.F.C.L.R.P./USP (Org.), Artigos do III Seminário de Pesquisa (Tomo II) do Programa de Pós-Graduação em Psicologia (pp. 101-107). Ribeirão Preto, SP: Autor.

Moreno, E., \& Pol, E. (1999). Nociones psicosociales para la intervención y la gestión ambiental (pp. 21-55). Barcelona: Publicacions de la Universitat de Barcelona.

Oliveira, Z. M. R. de, \& Rossetti Ferreira, M. C. (1993). O valor da interação criança-criança em creches no desenvolvimento infantil. Cadernos de Pesquisa, 87, 62-70.

Petrinovitch, I. (1979). Probabilistic functionalism - a conception of research method. American Psychologist, 34, 373-390.

Pinheiro, J. Q. (1997). Psicologia Ambiental: a busca de um ambiente melhor. Estudos de Psicologia, 2(2), 377-398.

Pol, E. (1996). La apropiación del espacio. In L. Íñiguez \& E. Pol (Orgs.), Cognición, representación y apropiación del espacio (pp. 45-62). Barcelona: Publications Universitat de Barcelona.

Proshansky, H. M., Ittelson, W. H., \& Rivlin, I. (1970). Environmental Psychology: man and his physical settings. Nova York: Holt, Rinehart \& Winston.

Rossetti-Ferreira. M. C., Amorim, K. S., \& Silva, A. P. S. (2000). Uma perspectiva teórico-metodológica para análise do desenvolvimento humano e do processo de investigação. Psicologia: Reflexão e Crítica, 13(2), 281-293.

Scheidt, R. J. (1981). Ecologically-valid inquiry: fait accompli? Human Development, 24, 225-228.

Sommer, B., \& Sommer, R. (1997). A practical guide to behavioral research: tools and techniques ( $4^{\underline{a}}$ ed.). Nova York: Oxford University Press.

Stokols, D. (1978). Environmental Psychology. Annual Review of Psychology, 29, 253-295.

Stokols, D. (1987). Conceptual strategies of Environmental Psychology. In D. Stokols \& I. Altman (Orgs.), Handbook of Environmental Psychology (vol. I, pp. 41-70). Nova York: John Wiley.

Stokols, D. (1992). Establishing and maintaining healthy environments - toward a social ecology of healthy promotion. American Psychologist, 47(1), 6-22.

Stokols, D. (1995). The paradox of Environmental Psychology. American Psychologist, 50, 821-837.

Tudge, J., Gray, J., \& Hogan, D. (1997). Ecological perspective in human development: a comparison of Gibson and Bronfenbrenner. In J. Tudge, M. Shanahan \& J. Valsiner (Orgs.), Comparisons in human development: understanding time and context (pp. 72-105). Nova York: Cambridge University Press.

Valsiner, J. (1987). Culture and the development of children's action - a culturalhistorical theory of developmental psychology. Nova York: Wiley.

Valsiner, J., \& Benigni, L. (1986). Naturalistic research and ecological thinking in the study of child development. Developmental Review, 6, 203-223.

Weisz, J. R. (1978). Transcontextual validity in developmental research. Child Development, 49, 1-12.

Mara I. Campos-de-Carvalho, doutora em Psicologia Experimental, Instituto de Psicologia da Universidade de São Paulo, é professora doutora de Psicologia Ambiental e do Desenvolvimento do Departamento de Psicologia e Educação da Faculdade de Filosofia, Ciências e Letras de Ribeirão Preto da Universidade de São Paulo. Endereço para correspondência: Departamento de Psicologia e Educação, Faculdade de Filosofia, Ciências e Letras de Ribeirão Preto, Universidade de São Paulo; Av. dos Bandeirantes, 3900; Ribeirão Preto, SP; 14040-901. Tel.: (16) 602-3661. Fax: (16) 633-5668. E-mail: mara@ffclrp.usp.br 